\title{
Obesidad central, sarcopenia y conductas sedentarias en el riesgo cardiovascular por score de Framingham y área total de placa carotídea
}

DOI: 10.17533/udea.penh.v22n2a02

PERSPECTIVAS EN NUTRICIÓN HUMANA

ISSN 0124-4108

Escuela de Nutrición y Dietética, Universidad de Antioquia. Medellín, Colombia

Vol. 22, N. ${ }^{\circ}$ 2, julio-diciembre de 2020, pp. 139-150.
Artículo recibido: 6 de septiembre de 2019

Aprobado: 25 de noviembre de 2020

Lorena Viola'; Néstor H. García; Hernán A. Pérez; Luis J. Armando4; Mariana N. Carrillo5;

Sonia E. Muñoz ${ }^{6}$; Laura R. Aballay ${ }^{7 *}$

\section{-- -Resumen}

Antecedentes: las enfermedades cardiovasculares son la principal causa de morbimortalidad mundial. La obesidad, sarcopenia, actividad física insuficiente y las conductas sedentarias impactan de manera sinérgica en el riesgo cardiovascular. Objetivo: evaluar el riesgo cardiovascular en relación con la actividad física, las conductas sedentarias y la composición corporal. Materiales y métodos: estudio observacional transversal de 95 personas adultas de ambos sexos. Se determinó el riesgo cardiovascular mediante el score de Framingham y el score de Framingham corregido por área total de placa aterosclerótica; la composición corporal, por antropometría, bioimpedancia y dinamometría como indicador indirecto; y la actividad física y las conductas sedentarias, por cuestionario validado. Se condujeron análisis descriptivos, de correlación y asociación con un $95 \%$ de confianza. Resultados: el $95 \%$ de las mujeres y el $98 \%$ de los varones presentaron riesgo cardiovascular elevado; el $51,5 \%$, obesidad; el $95,5 \%$, obesidad central; y el 47,3\%, fuerza muscular disminuida.

1 Licenciada en Nutrición. Centro de Investigaciones en Nutrición Humana (CenINH), Escuela de Nutrición. Facultad de Ciencias Médicas. Universidad Nacional de Córdoba. Bv. de la Reforma S/N, CP 5016, Córdoba, Argentina. violalorena@gmail.com

2 Doctor en Medicina y Cirugía. Instituto de Investigaciones en Ciencias de la Salud (INICSA), Universidad Nacional de Córdoba, CONICET, Facultad de Ciencias Médicas, Córdoba, Argentina. nestorhgarcia11@gmail.com

3 Especialista en Medicina Interna. Blossom DMO, Córdoba, Argentina. hperez05@gmail.com

4 Especialista en Cardiología. Blossom DMO, Córdoba, Argentina. luisjosearmando@gmail.com

5 Licenciada en Nutrición. Instituto de Investigaciones en Ciencias de la Salud (INICSA), Universidad Nacional de Córdoba, CONICET, Facultad de Ciencias Médicas, Córdoba, Argentina. mariana.n.carrillo@gmail.com

6 Doctora en Ciencias Biológicas. Instituto de Investigaciones en Ciencias de la Salud (INICSA), Universidad Nacional de Córdoba, CONICET, Facultad de Ciencias Médicas, Córdoba, Argentina. sonian2t@hotmail.com

$7^{\star} \quad$ Autor de correspondencia. Doctora en Ciencias de la Salud. Centro de Investigaciones en Nutrición Humana (CenINH), Escuela de Nutrición. Facultad de Ciencias Médicas. Universidad Nacional de Córdoba. Bv. de la Reforma S/N, CP 5016, Córdoba, Argentina. Teléfono: (0351) 535-3687. laballay@fcm.unc.edu.ar

Cómo citar este artículo: Viola L, García NH, Pérez HA, Armando LJ, Carrillo MN, Muñoz SE, Aballay LR. Obesidad central, sarcopenia y conductas sedentarias en el riesgo cardiovascular por Score Framingham y área total de placa carotídea. Perspect Nutr Humana. 2020;22:139-50. DOI: 10.17533/udea.penh.v22n2a02 
Se observaron asociaciones positivas significativas entre riesgo cardiovascular y circunferencia de cintura (rho $=0,26$; $p=0,024$ ). No hubo asociación significativa entre la fuerza muscular y el riesgo cardiovascular (rho=-0,21; $p=0,065$ ). La conducta sedentaria tuvo un efecto promotor del riesgo cardiovascular $(\mathrm{OR}=3,9 ; \mathrm{p}=0,033)$. Conclusiones: la obesidad central y permanecer más de 6/h día en posición sedente son factores asociados al riesgo cardiovascular.

Palabras clave: aterosclerosis coronaria, obesidad central, sarcopenia, actividad física, conductas sedentarias.

\section{Central Obesity, Sarcopenia, and Sedentary Behaviors Related to Cardiovascular Risk by Framingham Score and Total Carotid Plaque Area}

\section{Abstract}

Background: Cardiovascular diseases are the principal cause of morbidity and mortality worldwide. Obesity, sarcopenia, insufficient physical activity, and sedentary behaviors synergistically impact cardiovascular risk. Objective: Evaluate cardiovascular risk in relation to physical activity, sedentary behaviors, and body composition. Materials and Methods: Cross-sectional observational study in 95 total males and females. Cardiovascular risk was determined using the Framingham score, which corrects for total area of atherosclerotic plaque. Risk was also determined using body composition, anthropometry, bioimpedance and dynamometry as indirect indicators, physical activity, sedentary behaviors, and a validated questionnaire. Descriptive, correlation and association analyses were conducted with $95 \%$ confidence. Results: $95 \%$ of women and $98 \%$ of men presented with an elevated cardiovascular risk; $51.5 \%$ with obesity, $95.5 \%$ central obesity, and $47.3 \%$ with diminished muscular strength. Significant positive associations were observed between cardiovascular risk and waist circumference (rho $=0.26$; $\mathrm{p}=0.024$ ). There was no significant association between muscle strength and cardiovascular risk (rho $=-0.21$, $p=0.065)$. Sedentary behavior increased cardiovascular risk $(O R=3.9 ; p=0.033)$. Conclusions: Central obesity and staying more than six hours per day in a sitting position are factors associated with cardiovascular risk.

Keywords: Coronary atherosclerosis, central obesity, sarcopenia, physical activity, sedentary behaviors.

\section{INTRODUCCIÓN}

Las enfermedades cardiovasculares (ECV) son la principal causa de mortalidad y morbilidad mundial, pero su incidencia podría reducirse mediante un cambio en el estilo de vida (1). La inflamación crónica de bajo grado es un mecanismo fisiopatológico subyacente en enfermedades crónicas, entre ellas la obesidad, la sarcopenia y las ECV (2). En la fisiopatología de la ECV, la inflamación persistente y no resuelta de la pared vascular se asocia con la aterosclerosis, un proceso que, en general, presenta alteraciones subclínicas 0 manifestaciones clínicas de la edad adulta (3). La aterosclerosis subclínica es un predictor de riesgo cardiovascular (RCV) (4), que puede detec- tarse con una estrategia costo-efectiva de forma no invasiva mediante la determinación del área total de la placa carotídea (ATP) por ultrasonido (5).

En relación con la contribución de la obesidad al $\mathrm{RCV}$, la presencia de lípidos ectópicos, obesidad central y sarcopenia parece tener mayor relevancia que el peso corporal total o el tejido adiposo periférico o subcutáneo en el proceso inflamatorio (6). La obesidad central se asocia con una alteración en el perfil secretor, tanto del tejido adiposo como del adipocito, con mayor producción de adipoquinas proinflamatorias (7), mientras que la sarcopenia, definida principalmente por baja fuerza muscular (FM) que se confirma con la presencia de baja cantidad de masa muscular, es 
una condición que contribuye a la acumulación de tejido adiposo, a las conductas sedentarias (CS) y a un bajo nivel de actividad física (AF) (8). En ese sentido, la AF insuficiente se ha considerado en forma clásica como un factor de riesgo para ECV; sin embargo, en los últimos años se evidenció la importancia de ponderar las CS en forma independiente del nivel de AF, debido a su aumento en el estilo de vida actual y su relación con la ECV (9). Estudios recientes sugieren que personas que permanecen en posición sedente más de 6-8 $\mathrm{h} /$ día presentan mayor riesgo de obesidad, ECV, diabetes y cáncer, independiente de su nivel de $\mathrm{AF}$, con biomarcadores de resistencia a la insulina e inflamación más elevados $(10,11)$.

Así, el propósito del presente estudio fue indagar la relación entre la $A F$ y las $C S$ con la presencia de obesidad, obesidad central y sarcopenia en el RCV de personas adultas de ambos sexos, participantes en un programa de prevención de ECV de la provincia de Córdoba, Argentina.

\section{MATERIALES Y MÉTODOS}

\section{Diseño del estudio}

Se realizó un estudio observacional, de corte transversal, con abordaje descriptivo y analítico.

\section{Diseño muestral}

El muestreo fue aleatorio e incluyó a todas las personas adultas de ambos sexos residentes en la provincia de Córdoba, Argentina, que asistían a consulta médica cardiológica durante el período 2015-2016 y participaban en el programa de prevención de riesgo cardiovascular de la institución Blossom. Del total de 120 personas incluidas en el programa, 110 personas $(91,6 \%)$ proporcionaron su conformidad para participar en la investigación. Se excluyeron los participantes que no asistieron a la entrevista para estimar RCV o mediciones antro- pométricas y aquellos que informaron cualquier historia personal de enfermedad cardiovascular definida por infarto de miocardio previo o revascularización coronaria/periférica o cualquier síntoma actual potencialmente sugestivo de angina, definido por autoinforme de dolor torácico, presión torácica, opresión torácica, accidente cerebrovascular e insuficiencia renal crónica, lo que dejó finalmente constituida la muestra por 95 participantes que cumplieron los criterios de inclusión y completaron el estudio.

\section{Variables}

Se indagó sobre variables demográficas (sexo, edad) y de estilo de vida (nivel de actividad física y conductas sedentarias). Se determinó el riesgo cardiovascular mediante score de Framingham (SF) y SF corregido por área total de placa aterosclerótica. Se realizaron mediciones antropométricas (peso, talla, circunferencia de cintura), el porcentaje de grasa corporal por bioimpedancia y se utilizó la dinamometría como indicador indirecto de FM. Se recolectó, a partir de las historias clínicas, la presencia de factores de riesgo cardiovascular (diabetes, hipertensión y hábito tabáquico).

\section{Fuentes de información y proceso de recolec- ción de datos}

Se utilizaron fuentes de información secundaria (historias clínicas) y mediante profesionales entrenados, médicos especialistas y licenciados en nutrición se realizaron las mediciones de área de placa carotídea y antropometría y de estilos de vida, respectivamente. Las determinaciones y mediciones se obtuvieron durante una visita al servicio de cardiología en la institución Blossom de los pacientes pertenecientes al programa de prevención de riesgo cardiovascular. 


\section{Evaluación del riesgo cardiovascular}

El RCV se estimó por el SF y el SF corregido por área total de placa aterosclerótica carotídea (SFP). Para SF se utilizó una puntuación por factores de RCV según sexo, que permiten predecir el riesgo general de ECV a 10 años (12). El SF considera en su cálculo tanto características no modificables (edad, sexo) como factores de riesgo modificables (tabaquismo, dislipemia 0 IMC, diabetes e hipertensión).

El SFP considera, además de la puntuación de riesgo según $\mathrm{SF}$, el valor incremental de la combinación de la medición del área total de placa carotídea, con la probabilidad posterior a la prueba para la clasificación de RCV (5). El área total de la placa carotídea se midió utilizando un escáner de ultrasonido dúplex de alta resolución TL HDI 5000. La placa se definió como un engrosamiento local de la íntima $>1 \mathrm{~mm}$ de espesor. Las mediciones se realizaron en vistas longitudinales ampliadas de cada placa observada en las arterias carótidas comunes, internas y externas, derecha e izquierda. La suma de las áreas transversales de todas las placas vistas entre la clavícula y el ángulo de la mandíbula fueron tomadas como placa total carotídea. El área total de la placa se dividió entre dos y este valor se utilizó para el análisis posterior a la prueba (5).

Para clasificar SF y SFP, los individuos fueron divididos en tres grupos según el riesgo de presentar una ECV en los próximos 10 años: bajo riesgo $(\leq 10 \%)$, riesgo intermedio $(10-20 \%)$ y riesgo alto o elevado (> $20 \%$ ) (12).

\section{Evaluación nutricional y de la composición corporal}

El estado nutricional se valoró por medio del IMC; la adiposidad central, mediante la circunferencia de cintura (CC); la composición corporal, por medio del porcentaje de grasa corporal (GC) por bioimpedancia; y la FM, por dinamometría. Se determinó IMC por fórmula peso/talla². El peso (kg) se midió en balanza OMRON HBF-510LA; con la persona sin calzado y con ropa liviana. La talla $(\mathrm{m})$ se determinó con tallímetro de pared marca Seca escala 0 a $200 \mathrm{~cm}$, con talones juntos; cabeza, hombros y glúteos erguidos. La medición de la CC $(\mathrm{cm})$ se realizó con cinta antropométrica metálica Lufkin, con el abdomen descubierto, en posición erecta y relajada, en el punto medio entre el reborde costal y la cresta ilíaca. EI IMC y la CC se ajustaron a las técnicas de medición y los criterios de clasificación de la Organización Mundial de la Salud (OMS) (13).

La GC (\%) se estimó con un analizador OMRON HBF-306 de grasa corporal, con un ayuno previo del sujeto, de pie y sin haber realizado ejercicio físico previamente; se consideraron como valores deseables menos de 30 y $20 \%$ en mujeres y varones respectivamente según criterios de Bray et al., 1988 (14). La FM (kg) se midió con el sujeto de pie y ejerciendo la máxima presión palmar sobre el instrumento, utilizando dinamómetro Smedley Baseline 12-0286; se consideraron valores deseables aquellos mayores a $20 \mathrm{~kg}$ en mujeres y de $32 \mathrm{~kg}$ en varones (15).

\section{Actividad física y conductas sedentarias}

Se utilizó el cuestionario internacional de actividad física (IPAQ, 2003) propuesto por la OMS, para relevar el nivel de AF y las CS (16). La AF se clasificó en tres niveles: bajo, moderado y alto. Se consideró CS a la cantidad de tiempo (horas/día) que el individuo permanece en posición sentada 0 reclinada y en vigilia (17). 


\section{Análisis estadísticos}

Para la descripción de los datos, se calcularon estadísticos y medidas resumen de posición y de dispersión, estimando diferencia a través de la prueba t de Student. Se realizaron análisis de correlación entre las variables utilizadas para indicar el RCV y las variables antropométricas estudiadas, estimando los coeficientes de correlación de Pearson y sus respectivos valores de $p$.

El análisis confirmatorio se abordó ajustando modelos de regresión logística múltiple, se estimaron así los OR y sus intervalos de confianza asociados, ajustados por las variables de confusión (18). Todos los análisis se realizaron con el software estadístico STATA 14.0.

\section{Consideraciones éticas}

El presente trabajo fue aprobado por el Comité Institucional de Ética de la Investigación en Salud del Hospital Nacional de Clínicas, Córdoba, Argentina, e inscrito en el Registro Provincial de
Investigaciones en Salud (RePIS, N. ${ }^{\circ}$ Registro 118/12 y 119/12) del Ministerio de Salud de la Provincia de Córdoba. Cada individuo proporcionó su conformidad para participar en la investigación mediante un consentimiento informado.

\section{RESULTADOS}

El grupo estudiado estuvo conformado por 49 mujeres y 46 varones, $51,6 \%$ y $48,4 \%$ respectivamente, con una edad media $\pm \mathrm{DE}$ de $69,2 \pm 10,9$ años. El RCV por SF y SFP fue de $69,3 \%$ y $89,4 \%$ respectivamente, con diferencias significativas entre sexos solo para SF $(p=0,02)$. Respecto a los factores de RCV para SF y SFP, el $84,2 \%$ presentó hipertensión arterial; el $49,4 \%$, diabetes mellitus; el $51,5 \%$, obesidad; y el $50,5 \%$ era un grupo de fumadores actuales o exfumadores, con un área total de la placa carotídea media (DE) de 124,1(102,1) mm²; la proporción de varones fumadores o exfumadores fue significativamente mayor que la de mujeres $(p<0,01)$ (Tabla1).

Tabla 1. Riesgo cardiovascular y presencia de factores de riesgo cardiovascular de los participantes del estudio, Córdoba 2015-2016

\begin{tabular}{lcccc}
\hline Variables & Mujeres & Varones & Total & Valor $\mathbf{p}$ \\
\hline & $(\mathrm{n}=49)$ & $(\mathrm{n}=46)$ & $(\mathrm{n}=95)$ & \\
\hline RCV elevado por SF & $\mathrm{n}(\%)$ & $\mathrm{n}(\%)$ & $\mathrm{n}(\%)$ & $0,02^{*}$ \\
RCV elevado por SFP & $31(63,2)$ & $42(91,3)$ & $73(69,3)$ & 0,95 \\
Hipertensión arterial & $47(95,9)$ & $45(97,8)$ & $92(87,4)$ & 0,07 \\
Diabetes & $39(79,5)$ & $41(89,1)$ & $80(84,2)$ & 0,91 \\
IMC $\left(\geq 30 \mathrm{~kg} / \mathrm{m}^{2}\right)$ & $24(48,9)$ & $23(50,0)$ & $47(49,4)$ & 0,46 \\
Fumador 0 ex fumador & $23(46,9)$ & $26(56,5)$ & $49(51,6)$ & $<0,01^{*}$ \\
Fumador actual & $11(22,4)$ & $37(80,4)$ & $48(50,5)$ & 0,61 \\
ATP $\left(\mathrm{mm}^{2}\right)$ & $2(4,1)$ & $4(8,7)$ & $6(6,3)$ & 0,61 \\
\hline
\end{tabular}

RCV: riesgo cardiovascular. SF: score de Framingham. SFP: score de Framingham corregido por área de placa carotídea. IMC: Índice de Masa Corporal. Media (DE). ATP: área total de placa aterosclerótica carotídea.

${ }^{*} T$-test para diferencia de proporciones estadísticamente significativas entre sexos $(\alpha=0,05)$. 
Con respecto al estado nutricional, el $88,4 \%$ de la población presentó exceso de peso (IMC $\geq$ $\left.25 \mathrm{~kg} / \mathrm{m}^{2}\right)$, y dentro de estos el $51,5 \%$, obesidad (IMC $\left.\geq 30 \mathrm{~kg} / \mathrm{m}^{2}\right)$. Por otra parte, con relación a la composición corporal, el 95,5\% presentó obesidad central (CC aumentada) y el 96,8 \% GC total elevada, mientras que el $47,3 \%$ de los individuos estudiados presentó FM disminuida. No se observaron diferencias estadísticamente significativas para ninguno de estos indicadores entre sexos (Tabla 2). El 70,5 \% reportó un bajo nivel de AF y el $64 \%$ permanecía en posición sedente más de $6 \mathrm{~h} /$ día. La media de equivalentes metabólicos de la AF fue de 543 equivalentes metabólicos/ semana y declararon pasar un promedio de 5,6 h/ día sentados. No se observaron diferencias significativas entre mujeres y varones (Tabla 3).

Tabla 2. Características antropométricas de los participantes del estudio, Córdoba 2015 - 2016

\begin{tabular}{|c|c|c|c|c|}
\hline Variables & Mujeres & Varones & Total & Valor $p$ \\
\hline & $(n=49)$ & $(n=46)$ & $(n=95)$ & \\
\hline & $\mathrm{n}(\%)$ & $\mathrm{n}(\%)$ & $\mathrm{n}(\%)$ & \\
\hline IMC $\left(\mathrm{kg} / \mathrm{m}^{2}\right) \operatorname{Media}(D E)$ & $30,5(6,0)$ & $31,6(5,6)$ & $31,1(5,8)$ & \\
\hline Normal & $8(16,3)$ & $3(6,2)$ & $11(11,6)$ & \\
\hline Sobrepeso & $18(36,7)$ & $17(36,9)$ & $35(36,8)$ & 0,369 \\
\hline Obesidad & $23(46,9)$ & $26(56,5)$ & $49(51,5)$ & \\
\hline$C C^{*}(\mathrm{~cm})$ Media $(D E)$ & $99,2(11,5)$ & $110,5(15,7)$ & $104,8(14,8)$ & \\
\hline Deseable & $1(2,0)$ & $3(6,5)$ & $4(4,2)$ & $<0,001$ \\
\hline Aumentada & $6(12,2)$ & $11(23,9)$ & $17(17,9)$ & \\
\hline Muy aumentada & $42(85,7)$ & $32(69,6)$ & $74(77,9)$ & \\
\hline Grasa Corporal $^{\star}(\%)$ Media (DE) & $41,4(6,9)$ & $34,5(6,1)$ & $37,9(7,4)$ & $<0,001$ \\
\hline Deseable & $2(4,1)$ & $1(2,2)$ & $3(3,1)$ & \\
\hline Aumentada & $47(95,9)$ & $45(97,8)$ & $92(96,8)$ & \\
\hline Fuerza* (kg) Media (DE) & $19,6(5,1)$ & $33,4(9,4)$ & $26,5(10,2)$ & $<0,001$ \\
\hline Deseable & $25(51,0)$ & $25(54,3)$ & $50(52,6)$ & \\
\hline Baja-Intermedia & $24(48,9)$ & $21(45,2)$ & $45(47,4)$ & \\
\hline
\end{tabular}

* T-test para diferencia de medias estadísticamente significativas entre sexos $(p<0,05)$ IMC: Índice de Masa Corporal. CC: circunferencia de cintura.

Tabla 3. Actividad física y conducta sedente de los participantes del estudio, Córdoba 2015 - 2016

\begin{tabular}{lcccc}
\hline Variables & Mujeres & Varones & Total & Valor $p$ \\
\hline & $\begin{array}{l}(n=49) \\
X \pm D E\end{array}$ & $\begin{array}{l}(n=46) \\
X \pm D E\end{array}$ & $(n=95)$ & \\
\hline $\begin{array}{l}\text { Actividad Física } \\
\text { (METs/semana) }\end{array}$ & $513,2(786,4)$ & $578,1(945,7)$ & $543,6(860,3)$ & 0,720 \\
$\begin{array}{l}\text { Conducta Sedente } \\
\text { (horas sentado/día) }\end{array}$ & $5,2(3,5)$ & & & 0,335 \\
\hline
\end{tabular}

* METs: equivalentes metabólicos; $\mathrm{X} \pm \mathrm{DE}=$ promedio \pm desviación estándar. 
El análisis de correlación de Spearman indicó una asociación positiva significativa entre el RCV por SF y la CC (rho=0,26; $p=0,024)$. No hubo asociación significativa entre la FM y el RCV por SFP (rho=-0,04; $p=0,684$ ). El análisis de riesgo deter- minó que la CS tuvo un efecto promotor del RCV, y se observó que cuando las personas permanecían más de 6/h día en posición sedente tuvieron cuatro veces más probabilidad de presentar RCV elevado (OR=3,90; IC:1,11-13,67) (Tabla 4).

Tabla 4. Estimación de medidas de asociación en relación con la presencia de riesgo cardiovascular por score de Framingham, mediante modelos de regresión logística múltiple, ajustado por valor energético total, edad y sexo. Córdoba 2015 - 2016

\begin{tabular}{|c|c|c|c|}
\hline & OR (Odds Ratio) & IC 95\% & Valor $p$ \\
\hline Edad & 1,06 & $0,99-1,13$ & 0,052 \\
\hline \multicolumn{4}{|l|}{ Sexo } \\
\hline Masculino & 1 & & \\
\hline Femenino & 0,11 & $0,02-0,53$ & 0,006 \\
\hline \multicolumn{4}{|c|}{ Actividad física } \\
\hline Baja & 1 & & \\
\hline Moderada & 1,68 & $0,37-7,56$ & 0,494 \\
\hline Intensa & 1,08 & $0,14-8,13$ & 0,930 \\
\hline \multicolumn{4}{|c|}{ Conducta sedente } \\
\hline No sedente & 1 & & \\
\hline Sedente & 3,90 & $1,11-13,67$ & 0,033 \\
\hline \multicolumn{4}{|c|}{ Circunferencia de cintura } \\
\hline Adecuada & 1 & & \\
\hline Elevada & 0,99 & $0,95-1,04$ & 0,988 \\
\hline \multicolumn{4}{|c|}{ Fuerza muscular } \\
\hline Baja & 1 & & \\
\hline Intermedia & 2,60 & $0,36-18,44$ & 0,337 \\
\hline Normal & 1,16 & $0,30-4,46$ & 0,825 \\
\hline
\end{tabular}

* T-test para diferencia de medias estadísticamente significativas entre sexos $(p<0,05)$ IMC: Índice de Masa Corporal. CC: circunferencia de cintura.

\section{DISCUSIÓN}

El presente trabajo estudió 95 personas adultas de ambos sexos que asistían a un programa de prevención de riesgo cardiovascular de la provincia de Córdoba, Argentina. La mayoría de los individuos estudiados presentó RCV elevado, obesidad y obesidad central; además de un bajo nivel de AF y una CS elevada. Considerando los resultados de la última encuesta nacional de factores de riesgo realizada en el año 2018 en Argentina (19), en mayores de 18 años la prevalencia de exceso de peso (IMC>25 $\mathrm{kg} / \mathrm{m}^{2}$ ) fue de $66,1 \%$, prácticamente la mitad correspondía a sobrepeso $(33,7 \%$ y la otra mitad a obesidad $(32,4 \%)$, mientras que en el presente estudio, aunque no es el mismo grupo etario, es importante resaltar que casi la totalidad de las personas estudiadas (88\%) presentaron exceso de peso, porcentaje que incluye $51 \%$ de 
obesidad, con solo un $6 \%$ de los varones y $16 \%$ de las mujeres con IMC adecuado, lo que evidencia así el elevado riesgo de la población estudiada en comparación con la población general.

Con respecto a la obesidad central, estimada por la CC, solo el $2 \%$ de las mujeres y $6 \%$ de los varones presentaron valores normales; se observó una asociación entre el RCV y la obesidad central (rho=0,26; $p=0,024)$. Estudios previos reconocen un mayor impacto del tejido adiposo visceral, en lugar del IMC o el tejido adiposo periférico o subcutáneo en el proceso inflamatorio $(20,21)$. La obesidad abdominal o central es un factor de riesgo establecido para ECV y mortalidad por todas las causas; se asocia con mayor producción de adipoquinas proinflamatorias y con una menor sensibilidad a la insulina $(6,21)$. El tejido adiposo, particularmente el visceral, se considera un marcador de lípidos ectópicos, secretor de compuestos proinflamatorios que contribuyen a la injuria vascular, resistencia insulínica y aterogénesis $(20,21)$, lo que se considera de gran relevancia en la población estudiada, pues casi la totalidad $(95,5 \%)$ presentó obesidad central.

Con respecto a la presencia de sarcopenia, el $47 \%$ de la población presentó valores de FM disminuida, valor superior al hallado en otros estudios en los que las tasas de prevalencia se extienden de 2 a 34 \% según el criterio diagnóstico utilizado (22).

Si bien en el presente estudio no se observó asociación significativa entre la FM y el RCV, es conocido que la FM se asocia inversamente con el riesgo de mortalidad en diversas patologías agudas y crónicas (23). Por lo tanto, el RCV aumentado en individuos con baja FM es motivo de interés en los últimos años, en particular en el grupo estudiado, cuya edad promedio fue de 69 años y la presencia de baja FM afectó a casi la mitad de la población $(8,24)$.

Además, es importante considerar los hallazgos en el presente estudio -en el que se combina un alto porcentaje de personas que presentaron obesidad y baja FM-, pues son respaldados por evidencias recientes sobre las consecuencias del exceso de adiposidad corporal y la acumulación de lípidos ectópicos en el tejido muscular, que podrían favorecer la disfunción muscular y la disminución de la FM (25). Así, estas características, y en conjunto con la obesidad, son consideradas síndromes interconectados que se agravan mutuamente (26), que se asocian con trastornos metabólicos y con un mayor riesgo de ECV (25).

Con respecto a la $A F$, el presente estudio no encontró asociación significativa con el RCV. Sin embargo, es importante considerar que los mecanismos involucrados en la obesidad y la falta de FM comprenden el aumento del tejido adiposo ectópico, la infiltración grasa en el músculo esquelético, la inflamación crónica de bajo grado, la disfunción mitocondrial y la menor fuerza y función muscular, que indirectamente limitan la realización de AF adecuada y promueven las CS, lo que nuevamente aumenta la adiposidad corporal, y retroalimenta el problema (24).

Por último, una limitación que se debe considerar en el presente estudio es la evaluación de AF y CS mediante autoinforme, que, por lo tanto, está sujeta al sesgo del recuerdo. La mayoría de los individuos (70\%) presentó un nivel de AF bajo 0 insuficiente y solo el $8 \%$, intenso. No fue posible evaluar en este grupo la función protectora de la AF según su volumen y tipo, que se consideran adecuados para producir respuestas favorables en la prevención del RCV. El ejercicio físico de alta intensidad produciría efectos positivos sobre el estrés celular y las señales metabólicas necesa- 
rias para la biogénesis mitocondrial; también, en la reducción de la adiposidad corporal (27). En ese sentido, incrementar el nivel de AF, respetando dosis e intensidad apropiadas, se considera una herramienta terapéutica eficaz. En el presente estudio, las CS se asociaron con el RCV elevado, por lo que adquieren relevancia debido a las características de riesgo de la población. Las CS son cada vez más universales y motivadas por el entorno económico, social y físico, lo que se puede observar en el presente estudio en el que la media de CS al día fue de 5,6 horas y los equivalentes metabólicos (METs) de la AF reportada fueron de 543 METs/semana, inferiores al punto de corte de 600 METs que indica nivel de actividad física bajo. Es conocido que las CS en conjunto con la obesidad y el bajo nivel de AF se asocian con un mayor RCV (28); sin embargo, estudios recientes sugieren que las personas sedentarias presentan un perfil metabólico con biomarcadores de resistencia a la insulina e inflamación más elevados, independientemente de la AF realizada (10).

En el presente estudio se identificó un umbral de $6 /$ día de CS, valor por encima del cual aumenta el RCV, en concordancia con estudios previos, en los cuales más de 6-8 h/día total de CS y 3-4 h/día de ver televisión aumentaron el riesgo de mortalidad por todas las causas y ECV $(11,29)$. Incluso entre las personas que cumplen con las pautas de AF adecuada, las CS excesivas puede tener implicaciones metabólicas y pronósticos adversos, particularmente en adultos mayores, y contribuir al sobrepeso y a la obesidad por medio del aumento de la ingesta de energía y la disminución del gasto energético (30).

Se han sugerido mecanismos biológicos para explicar las asociaciones independientes de las CS, en particular para las enfermedades cardiometabólicas, mediante los efectos sobre el metabolismo de los lípidos y la glucosa en los grandes músculos esqueléticos involucrados en la postura y en la hemodinámica vascular (11). La pérdida de la estimulación contráctil local inducida al sentarse conduce a la reducción de la actividad de la lipoproteinlipasa del músculo esquelético, que se encuentra involucrada en la captación de triglicéridos, la producción de colesterol HDL y el metabolismo de la glucosa (31). Así mismo, estudios de laboratorio han demostrado los efectos beneficiosos de interrumpir las CS sobre el metabolismo de la glucosa e insulina posprandiales, y en menor medida en los biomarcadores cardiovasculares clásicos, como triglicéridos y colesterol (32).

En la población estudiada, el comportamiento sedentario representó un factor de RCV independiente del nivel de AF, ya que no se encontró asociación significativa entre RCV y AF, pero sí se evidenció cuatro veces más posibilidades de presentar alto RCV con elevada CS. Así, en el presente estudio se observa la interacción entre las CS y el aumento de la masa grasa visceral, en concordancia con estudios recientes, en los que se destaca la sinergia entre los factores del estilo de vida, la composición corporal y el RCV $(24,33)$.

Las principales debilidades detectadas en el presente estudio se relacionan con el reducido tamaño muestral, la técnica de entrevista que apela al recuerdo y la exactitud de las técnicas antropométricas para medición de las variables. Se consideran fortalezas del estudio la visión integrada de la salud en adultos mayores que contempla, además de mediciones de riesgo cardiovascular (área de placa carotídea y score Framingham), aspectos del estilo de vida potencialmente modificables como la alimentación, la actividad física, el tejido graso y la FM. 
En conclusión, los datos del presente estudio confirman observaciones previas en las cuales la obesidad central y las conductas sedentarias son factores asociados al riesgo cardiovascular. El equipo profesional debería implementar estrategias integrales en salud tendientes a mejorar la composición corporal y disminuir el tiempo de permanencia en posición sedente, para revertir estas condiciones; adicionalmente, promover la formación específica de recursos humanos que deberían buscar estos objetivos.

\section{CONFLICTO DE INTERESES}

Se declara que el manuscrito no contiene material protegido por derecho de reproducción ni genera conflictos de intereses.

\section{CONTRIBUCIÓN DE LOS AUTORES}

Se declara que todos los autores tienen contribución equitativa en el desarrollo de la investigación y la escritura del manuscrito.

\section{AGRADECIMIENTOS}

El presente estudio fue apoyado por subsidio a Proyectos de Investigación Plurianual (PIP N. ${ }^{\circ} 11220120100205$ CO) del Consejo Nacional de Investigación Científica y Tecnológica (CONICET). La autora Lorena Viola recibió apoyo financiero de una beca otorgada por el Ministerio de Educación de la Nación Argentina (PROMED 91/2015). Se agradece la subvención institucional sin restricciones de la institución Blossom DMO, Córdoba, Argentina.

\section{- -Referencias}

1. OMS. Enfermedades cardiovasculares. 2017. [Internet]. [Citado julio de 2019]. Disponible en: https://www.who.int/es/newsroom/fact-sheets/detail/cardiovascular-diseases-(cvds)

2. Collins KH, Herzog W, MacDonald GZ, Reimer RA, Rios JL, Smith IC, et al. Obesity, metabolic syndrome, and musculoskeletal disease: Common inflammatory pathways suggest a central role for loss of muscle integrity. Front Physiol. 2018;23(9):112. https://doi.org/10.3389/fphys.2018.00112

3. Gregor MF, Hotamisligil GS. Inflammatory mechanisms in obesity. Annu Rev Immunol. 2011;29:415-45. https://doi.org/10.1146/annurev-immunol-031210-101322

4. Inaba Y, Chen JA, Bergmann SR. Carotid plaque, compared with carotid intima-media thickness, more accurately predicts coronary artery disease events: A meta-analysis. Atherosclerosis. 2012;220:128-33. https://doi.org/10.1016/j.atherosclerosis.2011.06.044

5. Perez HA, Garcia NH, Spence JD, Armando LJ. Adding carotid total plaque area to the Framingham risk score improves cardiovascular risk classification. Arch Med Sci. 2016;12(3):513-20. https://doi.org/10.5114/aoms.2016.59924

6. Suárez-Carmona W, Sánchez-Oliver AJ, González-Jurado JA. Fisiopatología de la obesidad: perspectiva actual. Rev Chil Nutr. 2017;44(3):226-33. https://doi.org/10.4067/S0717-75182017000300226

7. Blüher M, Mantzoros CS. From leptin to other adipokines in health and disease: Facts and expectations at the beginning of the 21st century. Metabolism. 2015;64(1):131-45. https://doi.org/10.1016/j.metabol.2014.10.016

8. Cruz-Jentoft AJ, Bahat G, Bauer J, Boirie Y, Bruyère O, Cederholm T, et al. Writing group for the european working group on sarcopenia in older people 2 (EWGSOP2), and the extended group for EWGSOP2.Sarcopenia: Revised European consensus on definition and diagnosis. Age Ageing. 2019;48(1):16-31. https://doi.org/10.1093/ageing/afy169 
9. Biswas A, Oh PI, Faulkner GE, Bajaj RR, Silver MA, Mitchell MS, et al. Sedentary time and its association with risk for disease incidence, mortality, and hospitalization in adults: A systematic review and meta-analysis. Ann Intern Med. 2015;162(2):12332. https://doi.org/10.7326/M14-1651

10. Dickins KA, Buchholz SW, Rivero T, Miller C. A review of reviews: Sedentary behaviour and cardiovascular disease specific to older people. Int J Older People Nurs. 2018;13(4):e12211. https://doi.org/10.1111/opn.12211

11. Patterson R, McNamara E, Tainio M, de Sá TH, Smith AD, Sharp SJ, et al. Sedentary behaviour and risk of all-cause, cardiovascular and cancer mortality, and incident type 2 diabetes: A systematic review and dose response meta-analysis. Eur $\mathrm{J}$ Epidemiol. 2018;33(9):811-29. https://doi.org/10.1007/s10654-018-0380-1

12. D'Agostino RB, Vasan RS, Pencina MJ, Wolf PA, Cobain M, Massaro JM, et al. General Cardiovascular risk profile for use in primary care the framingham heart study. Circulation. 2008;117(6):743-53. https://doi.org/10.1161/CIRCULATIONAHA.107.699579

13. OMS. Obesity: Preventing and managing the global epidemic: Report of a WHO consultation on obesity, Geneva 1997. [Internet]. [Citado julio de 2019]. Disponible en: https://apps.who.int/iris/handle/10665/63854

14. Bray G, Bouchard C, James WPT. Definitions and proposed current classifications of obesity. En: Bray G, Bouchard C, James WPT (edits). Handbook of obesity. Nueva York: MarcekDekker; 1988, pp. 31-40.

15. Alley DE, Shardell MD, Peters KW, McLean RR, Dam TT, Kenny AM, et al. Grip strength cutpoints for the identification of clinically relevant weakness. J Gerontol A Biol Sci Med Sci. 2014;69(5):559-66. https://doi.org/10.1093/gerona/glu011

16. Craig CL, Marshall AL, Sjöström M, Bauman AE, Booth ML, Ainsworth BE et al. International physical activity questionnaire: 12-country reliability and validity. Med Sci Sports Exerc. 2003;35(8):1381-95. https://doi.org/10.1249/01.MSS.0000078924.61453.FB

17. Pate R, O'Neill J, Lobelo F. The evolving definition of "sedentary". Exerc Sport Sci Rev. 2008;36:173. https://doi.org/10.1097/JES.0b013e3181877d1a

18. Margaretts BM, Nelson M. Design concepts in nutritional epidemiology (2. ${ }^{a}$ ed.). New York: Oxford University Press; 2007, 471 pp.

19. Encuesta nacional de factores de riesgo, 4.a Edición. Dirección Nacional de Promoción de la Salud y Control de Enfermedades Crónicas No Transmisibles. Ministerio de Salud y Desarrollo Social de la Nación. Argentina, 2018. [Internet]. [Citado julio de 2019]. Disponible en: https://www.indec.gob.ar/ftp/cuadros/publicaciones/enfr_2018_resultados_definitivos.pdf

20. Ferrucci L, Fabbri E. Inflammageing: Chronic inflammation in ageing, cardiovascular disease, and frailty. Nat Rev Cardiol. 2018;15(9):505-22. https://doi.org/10.1038/s41569-018-0064-2

21. Suárez-Carmona W; Sánchez-Oliver AJ. Índice de masa corporal: ventajas y desventajas de su uso en la obesidad. Relación con la fuerza y la actividad física. Nutr Clin Med. 2018;XII(3):128-39. https://doi.org/10.7400/NCM.2018.12.3.5067

22. Reijnierse EM, Trappenburg MC, Leter MJ, Blauw GJ, Sipila S, Sillanpaa E et al. The impact of different diagnostic criteria on the prevalence of sarcopenia in healthy elderly participants and geriatric outpatients. Gerontology. 2015;61(6):491-6. https://doi.org/10.1159/000377699

23. Jochem C, Leitzmann M, Volaklis K, Aune D, Strasser B. Association between muscular strength and mortality in clinical populations: A systematic review and meta-analysis. J Am Med Dir Assoc. 2019;20(10):1213-23. https://doi.org/10.1016/j.jamda.2019.05.015

24. Abete I, Konieczna J, Zulet MA, Galmés-Panades AM, Ibero-Baraibar I, Babio $\mathrm{N}$ et al. Association of lifestyle factors and inflammation with sarcopenic obesity: Data from the PREDIMED-Plus trial. J Cachexia Sarcopenia Muscle. 2019;10:974-8. https://doi.org/10.1002/jcsm.12442 


\section{Obesidad, sarcopenia, conductas sedentarias y riesgo cardiovascular}

25. Wannamethee SG, Atkins JL. Muscle loss and obesity: The health implications of sarcopenia and sarcopenic obesity. Proc Nutr Soc. 2015;74(4):405-12. https://doi.org/10.1017/S002966511500169X

26. Kalinkovich A, Livshits G. Sarcopenic obesity or obese sarcopenia: A cross talk between age-associated adipose tissue and skeletal muscle inflammation as a main mechanism of the pathogenesis. Ageing Res Rev. 2017;35:200-21. https://doi.org/10.1016/j.arr.2016.09.008

27. Capodaglio EM. Physical activity, tool for the prevention and management of chronic diseases. G Ital Med Lav Ergon. 2018;40(2):106-19. Disponible en: https://pubmed.ncbi.nlm.nih.gov/30480395/

28. Silfee V, Lemon S, Lora V, Rosal M. Sedentary behavior and cardiovascular disease risk factors among Latino adults. J Health Care Poor Underserved. 2017;28(2):798-811. https://doi.org/10.1353/hpu.2017.0075

29. Dunstan D, Barr E, Healy GN, Salmon J, Shaw JE, Balkau B. Television viewing time and mortality: The AusDiab study. Circulation. 2010;121(3):384-91. https://doi.org/10.1161/CIRCULATIONAHA.109.894824

30. Chomistek AK, Manson JE, Stefanick ML, Lu B, Sands-Lincoln M, Going SB, et al. Relationship of sedentary behavior and physical activity to incident cardiovascular disease: results from the Women's Health Initiative. J Am Coll Cardiol. 2013;61(23):2346-54. https://doi.org/10.1016/j.jacc.2013.03.031

31. Hamilton MT, Hamilton DG, Zderic TW. Role of low energy expenditure and sitting in obesity, metabolic syndrome, type 2 diabetes, and cardiovascular disease. Diabetes. 2007;56(11):2655-67. https://doi.org/10.2337/db07-0882

32. Stamatakis E, Ekelund U, Ding D, Hamer M, Bauman A, Lee IM. Is the time right for quantitative public health guidelines on sitting? A narrative review of sedentary behaviour research paradigms and findings. Br J Sports Med. 2019;5386):377-82. https://doi.org/10.1136/bjsports-2018-099131

33. Polyzos SA, Margioris AN. Obesidad sarcopénica. Hormonas. 2018;17(3):321-31. https://doi.org/10.1007/s42000-018-0049-x 